\title{
A COMBINATORIAL COMMUTATIVITY PROPERTY FOR RINGS
}

\author{
HOWARD E. BELL and ABRAHAM A. KLEIN
}

Received 24 August 2001

We study commutativity in rings $R$ with the property that for a fixed positive integer $n$, $x S=S x$ for all $x \in R$ and all $n$-subsets $S$ of $R$.

2000 Mathematics Subject Classification: 16U80.

1. Introduction. In [2], we discussed $P_{\infty}$-rings $R$, which were defined by the property that

$$
X Y=Y X
$$

for all infinite subsets $X, Y$ of $R$; and in an earlier paper [1], the first author discussed $P_{n}$-rings, defined by the property that (1.1) holds for all $n$-subsets $X, Y$ of $R$. For a fixed positive integer $n$, we now define a $Q_{n}$-ring to be a ring $R$ with the property that

$$
x S=S x \quad \forall x \in R, \forall n \text {-subsets } S \text { of } R \text {. }
$$

Clearly, every commutative ring is a $Q_{n}$-ring for arbitrary $n$; moreover, there exist badly noncommutative $Q_{n}$-rings, since every ring with fewer than $n$ elements is a $Q_{n}$ ring. Our purpose is to identify conditions which force $Q_{n}$-rings to be commutative or nearly commutative.

It is obvious that every $Q_{n}$-ring is a $P_{n}$-ring and every $P_{n}$-ring is a $P_{\infty}$-ring. We make no use of the results on $P_{n}$-rings in [1], and most of our results are of a different sort than those in [1]. However, a special case of the theorem on $P_{\infty}$-rings in [2] plays a crucial role in our study.

2. Preliminaries. We begin with some notation. Let $R$ be an arbitrary ring, not necessarily with 1 . The symbols $D, N, Z$, and $C(R)$ denote the set of zero divisors, the set of nilpotent elements, the center, and the commutator ideal, respectively; and $|R|$ denotes the cardinal number of $R$. For $Y$ being an element or subset of $R$, the symbols $C_{R}(Y), A_{\ell}(Y), A_{r}(Y)$, and $A(Y)$ denote the centralizer of $Y$ and the left, right, and twosided annihilators of $Y$. For $x, y \in R$, the set $L_{x, y}$ is defined to be $\{w \in R \mid x y=w x\}$.

We give three lemmas, the first of which is rather trivial and the other two of which are not.

LEMMA 2.1. Let $R$ be a $Q_{n}$-ring with $|R| \geq n$. Then

(i) for all $x \in R, x R=R x$ and $\left|A_{\ell}(x)\right|=\left|A_{r}(x)\right|$;

(ii) all idempotents of $R$ are central; 
(iii) $N$ is an ideal;

(iv) if $R$ is not commutative and $x \notin Z$, then $R \backslash\left(A_{\ell}(x) \cup C_{R}(x)\right)$ and $R \backslash\left(A_{r}(x) \cup\right.$ $\left.C_{R}(x)\right)$ are nonempty.

Proof. (i) is obvious; and if $e$ is idempotent, the fact that $e R=R e$ yields $e x=$ exe $=x e$ for all $x \in R$, so $e \in Z$. Moreover, (i) enables us to prove (iii) by adapting the standard proof that $N$ is an ideal in commutative rings. Finally, if $x \notin Z$ then $C_{R}(x)$ is a proper subgroup of $(R,+)$; and (i) implies that $A_{\ell}(x)$ and $A_{r}(x)$ are also proper subgroups of $(R,+)$. Since a group cannot be the union of two proper subgroups, (iv) is immediate.

LEMMA 2.2. If $R$ is an infinite $Q_{n}$-ring, then $R$ is commutative.

Proof. Since every $Q_{n}$-ring is a $P_{\infty}$-ring, we could simply invoke the theorem of [2], which states that every $P_{\infty}$-ring is either finite or commutative. However, the proof in [2] is long and involved, so we prefer to give a more elementary proof.

Let $R$ be a noncommutative $Q_{n}$-ring. We may assume that $R$ is not a $Q_{m}$-ring for any $m<n$. Since all $Q_{1}$-rings are commutative, $n>1$, and there exist $x \in R$ and an $(n-1)$-subset $H$ of $R$ such that $x H \neq H x$; and we may assume that $x H$ is not a subset of $H x$. We may also assume that $R \backslash H \neq \varnothing$, since otherwise $R$ is finite.

For any $a \in R \backslash H, x(H \cup\{a\})=(H \cup\{a\}) x$, so if we take $h \in H$ for which $x h \notin H x$, we have

$$
x h=a x .
$$

Since (2.1) holds for all $a \in R \backslash H$, it follows that for fixed $b \in R \backslash H, R \backslash H \subseteq b+A_{\ell}(x)$. Moreover, if $c \in A_{\ell}(x)$, then $x h=b x=(b+c) x$, so $b+c \notin H$. Therefore $R \backslash H=$ $b+A_{\ell}(x)$, hence $|R \backslash H|=\left|A_{\ell}(x)\right|$ and $\left|R \backslash A_{\ell}(x)\right|=|H|$. But since $A_{\ell}(x)$ is a proper subgroup of $R,\left|R \backslash A_{\ell}(x)\right| \geq\left|A_{\ell}(x)\right|$, that is, $|H| \geq|R \backslash H|$; and the finiteness of $H$ yields the finiteness of $R$.

LEMMA 2.3 (see [4]). If $R$ is a finite ring with $N \subseteq Z$, then $R$ is commutative.

In view of Lemma 2.2, we assume henceforth that $R$ is finite.

\section{Commutativity of $Q_{n}$-rings with 1}

THEOREM 3.1. If $R$ is any $Q_{n}$-ring with 1 such that $|R|>n$, then $R$ is commutative.

Proof. By Lemma 2.3, we need only to show that $N \subseteq Z$; and since $u \in N$ implies $1+u$ is invertible, it suffices to prove that invertible elements are central.

Suppose, then, that $x$ is a noncentral invertible element and $y \notin C_{R}(x)$. If $H$ is any $(n-1)$-subset of $R$ which excludes $y$, the condition $x(\{y\} \cup H)=(\{y\} \cup H) x$ yields $z \in H$ such that

$$
x y=z x .
$$

Since $x$ is invertible, there is a unique $z \in R$ satisfying (3.1); and we have shown that every (n-1)-subset contains either $y$ or $z$. But $|R \backslash\{y, z\}| \geq n-1$; therefore noncentral invertible elements cannot exist. 
The bound on $|R|$ in Theorem 3.1 is best possible, as the following example shows. The rings of this example were introduced by Corbas in [3].

EXAMPLE 3.2. Let $n=p^{2 k}$, where $p$ is prime and $k>1$. Let $\phi$ be a nonidentity automorphism of $G F\left(p^{k}\right)$. Let $R=G F\left(p^{k}\right) \times G F\left(p^{k}\right)$, with addition being componentwise and multiplication given by $(a, b)(c, d)=(a c, a d+b \phi(c))$. It is easily shown that $R$ is a ring with $|R|=n$ and $D=\left\{(0, b) \mid b \in G F\left(p^{k}\right)\right\}$; hence if $a \neq 0,(a, b)$ is invertible. Thus, if $a \neq 0,(a, b) R=R(a, b)=R$; and if $b \neq 0,(0, b) R=\left\{(0, b \phi(c)) \mid c \in G F\left(p^{k}\right)\right\}$ and $R(0, b)=\left\{(0, b c) \mid c \in G F\left(p^{k}\right)\right\}$, so that $(0, b) R=R(0, b)=D$. Thus, $R$ is a $Q_{n^{-}}$ ring. Obviously, $R$ is noncommutative and $(1,0)$ is a multiplicative identity element.

4. Commutativity of $Q_{n}$-rings: the general case. We begin this section with a nearcommutativity theorem, which is reminiscent of [1, Theorem 6].

THEOREM 4.1. If $n \leq 16$ and $R$ is any $Q_{n}$-ring, then $C(R)$ is nil.

Proof. Since every $Q_{k}$-ring is a $Q_{k+1}$-ring, we may assume that $n=16$. If $|R| \geq 16$, then $N$ is an ideal by Lemma 2.1(iii); and $R / N$ is a finite ring with no nonzero nilpotent elements, hence is commutative. If $|R|<16$, it follows easily from the WedderburnArtin structure theory that $C(R)$ is nil.

We proceed to our major commutativity theorems.

THEOREM 4.2. Let $n \geq 4$, and let $R$ be a $Q_{n}$-ring. If $|R|>2 n-2$, or if $n$ is even and $|R|>2 n-4$, then $R$ is commutative.

Proof. Let $R$ be a $Q_{n}$-ring which is not commutative, and let $x \notin Z$. Our aim is to show that $|R| \leq 2 n-2$ or $|R| \leq 2 n-4$; and since $n-1<2 n-4$, we may suppose that $|R| \geq n$. By Lemma 2.1(iv), there exists $y \in R \backslash\left(A_{r}(x) \cup C_{R}(x)\right)$. If $H$ is any $(n-1)$ subset which does not contain $y$, we have $x(\{y\} \cup H)=(\{y\} \cup H) x$; and since $x y \neq$ $y x$, there exists $z \in H$ such that $x y=z x$-that is, $H \cap L_{x, y} \neq \varnothing$. We have argued that any $(n-1)$-subset of $R$ must either contain $y$ or intersect $L_{x, y}$-a condition that cannot hold if $\left|R \backslash L_{x, y}\right| \geq n$; thus,

$$
|R| \leq\left|L_{x, y}\right|+n-1
$$

We now investigate $\left|L_{x, y}\right|$. If $w \in L_{x, y}$, then $L_{x, y}=w+A_{\ell}(x)$, hence $\left|L_{x, y}\right|=\left|A_{\ell}(x)\right|$. By Lemma 2.1(iv), $A_{\ell}(x) \neq R$, so $\left|L_{x, y}\right|=|R| / k$ for some $k \geq 2$. Substituting into (4.1) gives

$$
|R| \leq \frac{k}{k-1}(n-1) \leq 2 n-2 .
$$

Suppose now that $n$ is even. If $A_{\ell}(x)$ has index at least 3 in $(R,+),(4.2)$ yields $|R| \leq\lfloor 3(n-1) / 2\rfloor \leq 2 n-4$. Thus, we may assume that $\left|A_{\ell}(x)\right|=|R| / 2$ and show that $|R| \neq 2 n-2$.

Suppose, then, that $\left|A_{\ell}(x)\right|=n-1$, so that $\left|A_{r}(x)\right|=n-1$ by Lemma 2.1(i). Note that $A_{\ell}(x)$ is an $(n-1)$-subset not intersecting $L_{x, y}$, so $y$ must be in $A_{\ell}(x)$; and since 
$y \notin A_{r}(x), A_{\ell}(x) \neq A_{r}(x)$, so $A_{r}(x) x \neq\{0\}$. Now $x\left(y \cup A_{r}(x)\right)=\left(y \cup A_{r}(x)\right) x$ and therefore $A_{r}(x) x \subseteq\{x y, 0\}$; hence $A_{r}(x) x=\{0, x y\}$ is an additive subgroup of order 2. Therefore the map $\phi: A_{r}(x) \rightarrow A_{r}(x) x$ given by $w \mapsto w x$ has kernel of index 2 in $A_{r}(x)$. But $\left|A_{r}(x)\right|$ is odd, so we have a contradiction; hence $|R| \leq 2 n-4$.

As we will see later, the bounds on $|R|$ in Theorem 4.2 are best possible; however, under various restrictions, a smaller bound holds.

THEOREM 4.3. Let $n \geq 4$ and let $R$ be a $Q_{n}$-ring with $|R|>(3 / 2)(n-1)$. Then $R$ is commutative if one of the following is satisfied:

(i) $|R|$ is odd;

(ii) $(R,+)$ is not the union of three proper subgroups;

(iii) $N$ is commutative;

(iv) $R^{3} \neq\{0\}$.

Proof. Again we suppose that $R$ is not commutative and $x \notin Z$. Since $|R|>$ $(3 / 2)(n-1)>n$, the arguments in the proof of Theorem 4.2 show that $\left|A_{\ell}(x)\right|=$ $\left|A_{r}(x)\right|=|R| / 2$-a fact which proves that (i) implies commutativity of $R$.

Applying the first isomorphism theorem for groups shows that $|x R|=|R x|=2$; hence for any $u \in R \backslash A_{r}(x)$ and $v \in R \backslash A_{\ell}(x), x R=\{0, x u\}$ and $R x=\{0, v x\}$. Since $x R=R x$ by Lemma 2.1(i), it follows that if $y \in R \backslash\left(A_{\ell}(x) \cup A_{r}(x)\right)$, then $\{0, x y\}=$ $x R=R x=\{0, y x\}$ and therefore $y \in C_{R}(x)$. Thus $R=A_{\ell}(x) \cup A_{r}(x) \cup C_{R}(x)$, and we have proved that (ii) implies commutativity of $R$.

We now show that $x \in N$. Since $R$ is not commutative, it follows from Theorem 3.1 that $R$ does not have 1 , hence $R=D$; and if $x \notin N$, some power of $x$ is an idempotent zero divisor $e \neq 0$. Since $A_{\ell}(x) \subseteq A_{\ell}(e)$ and $A_{\ell}(e) \neq R$, we must have $A_{\ell}(x)=A_{\ell}(e)$ and similarly $A_{r}(x)=A_{r}(e)$. But $e$ is central by Lemma 2.1(ii), hence $A_{\ell}(x)=A_{r}(x)=$ $A(x) \subseteq C_{R}(x)$. Thus, if $y \notin A(x),\{0, x y\}=x R=R x=\{0, y x\}$ and $y$ is also in $C_{R}(x)$, contrary to the assumption that $x \notin Z$. But $x$ was an arbitrary noncentral element; hence, if there exist two noncommuting elements, both must be nilpotent. Thus (iii) forces commutativity of $R$.

To complete our proof, we show that our assumption that $R$ is not commutative forces $R^{3}=\{0\}$. For $x \notin Z$, the fact that $x \in N$ yields $A_{r}\left(x^{2}\right) \supsetneq A_{r}(x)$, so $A_{r}\left(x^{2}\right)=R$; hence $x^{2} R=R x^{2}=\{0\}$. If we choose $y \in R \backslash\left(A_{r}(x) \cup C_{R}(x)\right)$ and $w \in R \backslash\left(A_{\ell}(x) \cup\right.$ $C_{R}(x)$ ), then $y^{2} R=R y^{2}=\{0\}$; moreover, $\{0, x y\}=x R=R x=\{0, w x\}$, so $x y=w x$. Thus, $x R^{2}=x y R=w x R=\{w x y, 0\}=\left\{x y^{2}, 0\right\}=\{0\}$. If $z \in Z$, then $x+z \notin Z$ so $(x+z) R^{2}=\{0\}$; therefore $R^{3}=\{0\}$ as required.

We now give examples showing that the bounds on $|R|$ in Theorems 4.2 and 4.3 are best possible.

EXAMPLE 4.4. Let $R$ be the algebra over $G F(2)$ with basis $x, y, x^{2}$ and multiplication defined by $x y=x^{2}=y^{2}, 0=y x=x^{2} y=y x^{2}=x x^{2}=x^{2} x=x^{2} x^{2}$. Then $\left\{0, x^{2}\right\}=$ $A(R)$. It is easily verified that for any $u \notin A(R)$, the sets $A_{\ell}(u), A_{r}(u),\{w \in R \mid$ $\left.u w=x^{2}\right\}$ and $\left\{w \in R \mid w u=x^{2}\right\}$ all have 4 elements; hence for any 5-subset $S$ of $R$, $u S=S u=\left\{0, x^{2}\right\}$. Therefore $R$ is a $Q_{5}$-ring, and hence a $Q_{6}$-ring, with $|R|=8$. Thus, in general, neither bound in Theorem 4.2 can be improved. 
EXAMPLE 4.5. Let $R$ be the algebra over $G F(3)$ with basis $x, y, x^{2}$ and multiplication defined as in the previous example. An argument similar to the one above shows that $R$ is a $Q_{19}$-ring with $|R|=27$, so the bound $(3 / 2)(n-1)$ in Theorem 4.3 cannot be reduced.

5. Further results for small $n$. By definition all $Q_{1}$-rings are commutative, and it is easy to see that all $Q_{2}$-rings are commutative; and since there exist rings of order 4 which are not commutative, not all $Q_{5}$-rings are commutative. It is natural to ask: what is the largest $n$ such that all $Q_{n}$-rings are commutative? Our next theorem gives the answer.

THEOREM 5.1. If $n \leq 4$, all $Q_{n}$-rings are commutative.

PROoF. Since every $Q_{k}$-ring is a $Q_{k+1}$-ring, we may assume $n=4$. By Theorem 4.2 any counterexample $R$ would have $|R| \leq 4$; and since all rings of order less than 4 are commutative, we need only to consider rings of order 4 .

Suppose, then, that $R$ is a counterexample and $x$ and $y$ are noncommuting elements with $x y \neq 0$. Then $R=\{0, x, y, x+y\}$. Since idempotents are central, any of $x^{2}=x$, $x^{2}=y, x^{2}=x+y$ would force $x$ and $y$ to commute; hence $x^{2}=0$. It is now easily checked that the condition $x R=R x$ cannot hold.

Not surprisingly, a better result holds for rings with 1 .

THEOREM 5.2. If $n \leq 8$, then every $Q_{n}$-ring with 1 is commutative.

Proof. We may assume that $n=8$. Suppose that $R$ is a counterexample. By Theorem $3.1,|R| \leq 8$; and since all rings with 1 having fewer than 8 elements are commutative, $|R|=8$ and $R$ is indecomposable. Since idempotents are central, we therefore have no idempotents except 0 and 1 ; hence every element is either nilpotent or invertible. Since $u \in N$ implies $1+u$ is invertible, it follows from Lemma 2.3 that there exists a pair $x, y$ of noncommuting invertible elements. The group of units is not commutative and has at most 7 elements, hence is isomorphic to $S_{3}$. Thus, there exists a unique nonzero nilpotent element $u$, which by Lemma 2.3 is not central; and there is therefore an invertible element $w$ such that $u w \neq w u$. But in view of Lemma 2.1(iii), $w u$ and $u w$ are nonzero nilpotents, so we have a contradiction.

Theorem 5.2 is best possible; the ring of upper-triangular $2 \times 2$ matrices over $G F(2)$ is a $Q_{9}$-ring with 1 which is not commutative.

ACKNOWLEDGMents. The authors are grateful to Professor B. H. Neumann for suggesting that we study $Q_{n}$-rings. The first author was supported by the Natural Sciences and Engineering Research Council of Canada, Grant No. 3961.

\section{REFERENCES}

[1] H. E. Bell, A setwise commutativity property for rings, Comm. Algebra 25 (1997), no. 3, 989-998.

[2] H. E. Bell and A. A. Klein, A commutativity and finiteness condition for rings, in preparation.

[3] B. Corbas, Rings with few zero divisors, Math. Ann. 181 (1969), 1-7. 
[4] I. N. Herstein, A note on rings with central nilpotent elements, Proc. Amer. Math. Soc. 5 (1954), 620.

Howard E. Bell: Department of Mathematics, Brock University, St. Catharines, ONTARIO, CANADA L2S 3A1

E-mail address: hbe11@spartan.ac.brocku.ca

Abraham A. Klein: Sackler faculty of Exact Sciences, School of Mathematical Sciences, Tel AVIV University, Tel AVIV 69978, IsRAel

E-mail address: aak1ein@post.tau.ac.i1 


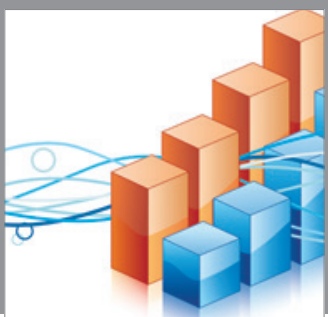

Advances in

Operations Research

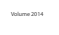

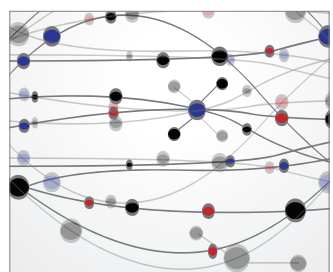

\section{The Scientific} World Journal
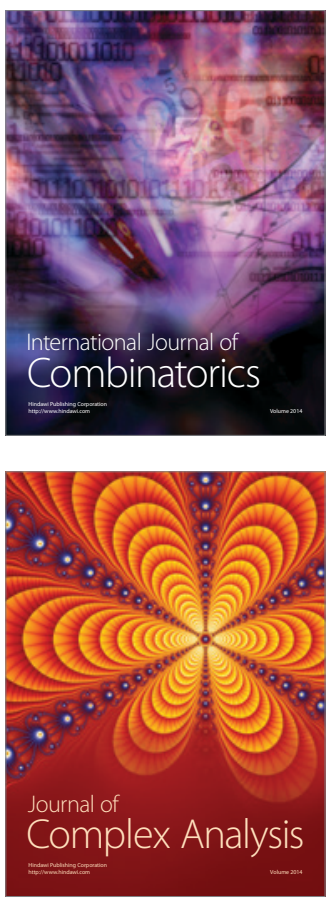

International Journal of

Mathematics and

Mathematical

Sciences
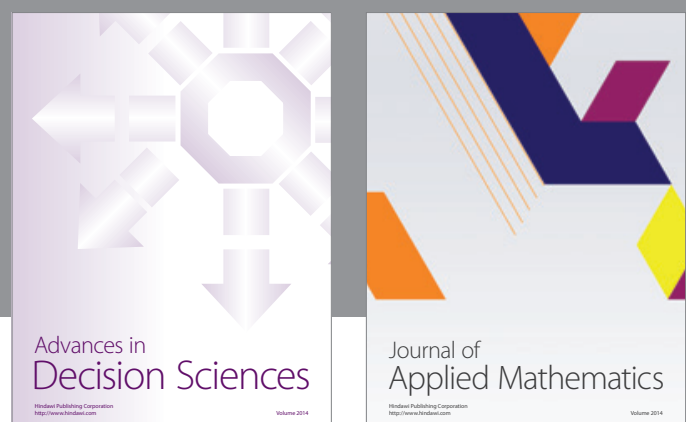

Journal of

Applied Mathematics
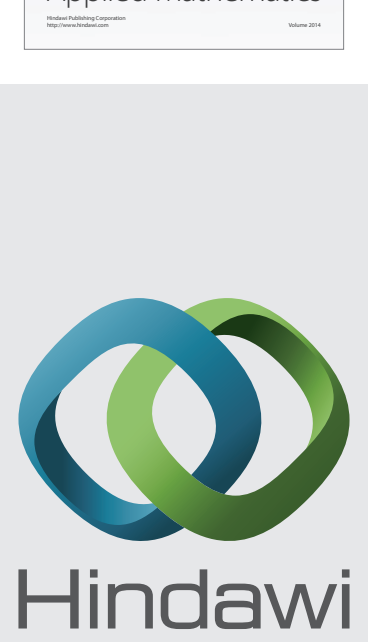

Submit your manuscripts at http://www.hindawi.com
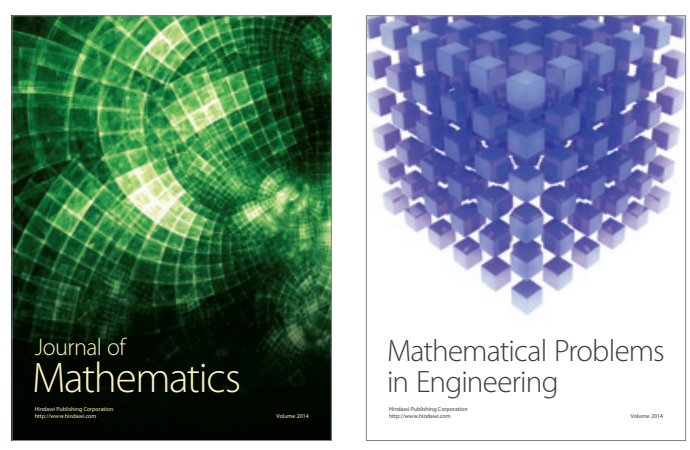

Mathematical Problems in Engineering
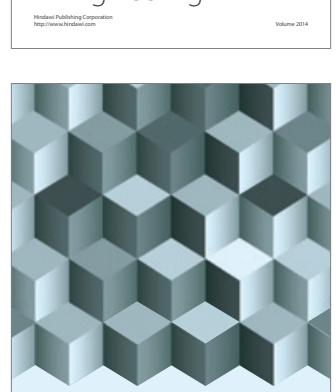

Journal of

Function Spaces
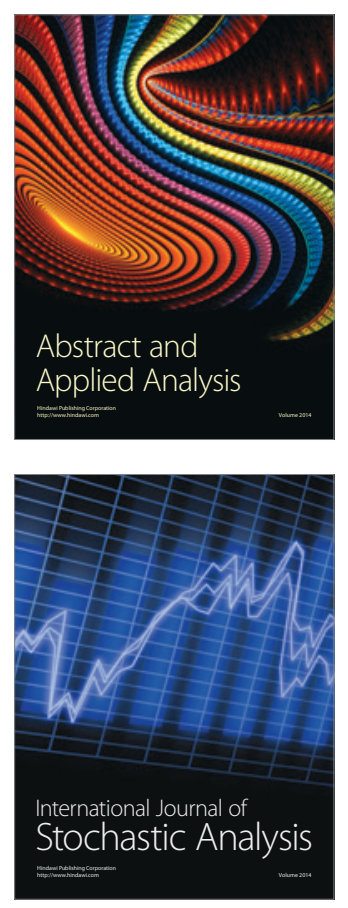

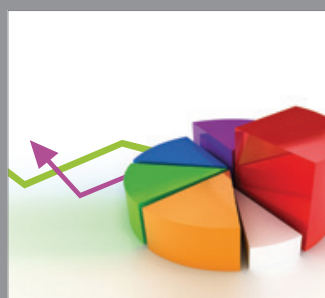

ournal of

Probability and Statistics

Promensencen
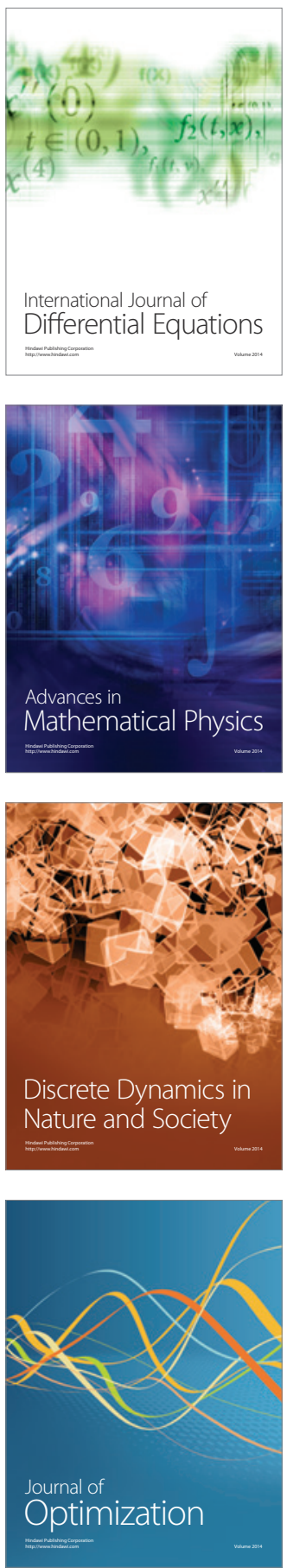